\title{
(2) OPEN ACCESS \\ Nicotine dependence of cigarette and heated tobacco users in Japan, 2019: a cross-sectional analysis of the JASTIS Study
}

\author{
Yan Kwan Lau (1) ,' Sumiyo Okawa, ${ }^{2}$ Rafael Meza 다, ${ }^{1}$ Kota Katanoda, ${ }^{3}$ \\ Takahiro Tabuchi ${ }^{2}{ }^{2}$
}

${ }^{1}$ Department of Epidemiology, University of Michigan School of Public Health, Ann Arbor, Michigan, USA

${ }^{2}$ Cancer Control Center, Osaka International Cancer Institute, Osaka, Japan

${ }^{3}$ Division of Cancer Statistics Integration, Center for Cancer Control and Information Services, National Cancer Center, Tokyo, Japan

\section{Correspondence to} Dr Takahiro Tabuchi, Cancer Control Center, Osaka International Cancer Institute, Osaka, 541-8567, Japan; tabuti-ta@mc.pref.osaka.jp

Received 15 September 2020 Revised 28 December 2020 Accepted 6 January 2021
D) Check for updates

(c) Author(s) (or their employer(s)) 2021. Re-use permitted under CC BY-NC. No commercial re-use. See rights and permissions. Published by BMJ.

To cite: Lau YK, Okawa S, Meza R, et al. Tob Control Epub ahead of print: [please include Day Month Year]. doi:10.1136/

tobaccocontrol-2020-056237

\section{ABSTRACT}

Objectives Japan is currently the biggest market of heated tobacco products (HTPs) in the world. Little is known about nicotine dependence among HTP users. Thus, the objective was to assess the association of type of tobacco use and time-to-first-use, a marker of nicotine dependence.

Methods A cross-sectional analysis of the 2019 data from an internet cohort study was conducted. The analytical sample consisted of 2147 current ( $\geq 1$ day use in the past 30 days) HTP and/or conventional cigarette users, aged 25+ years. Marginal structural binomial regression was used to estimate nicotine dependence prevalence ratios (PRs) for each category of tobacco use (exclusive daily cigarette, exclusive HTP ( $\geq 1$ day), dual HTP+daily cigarette, dual HTP+non-daily cigarette), relative to exclusive, non-daily cigarette smoking. Results Using a 5 min cut-off for time-to-first-use, the prevalence of nicotine dependence was higher among dual users of HTP and daily cigarettes (PR=1.38; 95\% $\mathrm{Cl}: 1.05$ to 1.82$)$ and exclusive, daily cigarette users ( $P R=1.48 ; 95 \% \mathrm{Cl}: 1.15$ to 1.91), relative to exclusive, non-daily cigarette users. However, nicotine dependence among exclusive HTP users, and dual HTP+non-daily cigarette users, did not differ from that of exclusive, non-daily cigarette users. When using 15 and $30 \mathrm{~min}$ cut-offs, all types of users, including exclusive HTP, had higher levels of nicotine dependence relative to exclusive, non-daily cigarette users.

Conclusions Regardless of HTP use, daily cigarette users had higher prevalence of nicotine dependence compared with non-daily cigarette users. Exclusive HTP users had similar (or potentially higher) dependence compared with exclusive, non-daily cigarette users. Longitudinal studies are needed to interrogate the public health implications of growing HTP use worldwide.

\section{INTRODUCTION}

Heated tobacco products (HTPs) are electronic devices that heat tobacco leaf and generate a nicotine-containing aerosol for inhalation. ${ }^{1}$ The Japanese National Health and Nutrition Survey from 2018 reported that $22.1 \%$ of male and $14.8 \%$ of female current tobacco users were heated tobacco users. ${ }^{2}$ IQOS, an HTP from Philip Morris, began its roll out in Japan in November 2014, and is reportedly accounting for $17.6 \%$ of the overall Japanese tobacco market sales (by product units) as of December 2019. ${ }^{3}$ By Philip Morris' own estimates, they currently have the largest global HTP market share with its core market in Japan. ${ }^{4}$ British
American Tobacco (glo series) and Japan Tobacco (Ploom series) introduced competing products soon after IQOS' initial introduction, and currently account for $19.6 \%{ }^{5}$ and $9 \%{ }^{6}$ of sales in the HTP category, respectively. Imperial Tobacco Japan released PULZE in October 2019.

Recent studies have shown that the introduction of HTP in Japan was likely responsible for the concurrent reduction in conventional cigarette consumption. ${ }^{7}$ However, the long-term public health impact of switching from conventional cigarettes to HTP remains to be determined. First, the extent of switching is unknown: are HTP users completely quitting conventional cigarettes, or do HTP users sustain the use of some level of conventional cigarette smoking; that is, dual use? ${ }^{9}$ HTPs have been described as a 'better alternative to cigarettes' and 'reduced risk product(s)' by tobacco companies. ${ }^{36}$ Indeed, Philip Morris filed an application with the US Food and Drug Administration (FDA) for IQOS to be classified as a modified risk tobacco product in the USA so that it could be marketed with claims of reduced risk and exposure relative to traditional cigarettes. ${ }^{10} 11$ Recently, the FDA granted IQOS permission to be marketed as a product with 'reduced exposure', but denied it to be marketed with 'reduced risk' claims considering the lack of evidence. ${ }^{11}$

Current research suggests that harmful and potentially harmful constituents (HPHCs) such as tobacco-specific nitrosamines (TNSAs) and volatile organic compounds, some of which are known carcinogens, were still found in mainstream aerosol of IQOS. ${ }^{12} 13$ Compared with conventional cigarettes, the levels of many HPHCs were lower. ${ }^{13}$ However, there were a number of HPHCs detected in IQOS emissions that had high levels, and some of these have unknown health risks. ${ }^{10}$ Therefore, additional studies are needed to better understand the health implications of HTP use. Independently, even if decreased exposure to HPHCs from switching could prove to be less harmful, dual use that sustains a low level of cigarette would still have deleterious health effects. ${ }^{14}$

Common to addictions of other substances, social and economic influences, gender, genetic predispositions, psychiatric disorders and behavioural/ sensory cues are some of the important determinants of tobacco addiction. ${ }^{15}$ In this study, however, we focus on nicotine dependence; to understand the potential interactions of HTP use and conventional cigarette smoking, information is needed 
on the degree of nicotine dependence. The level of nicotine measured in mainstream aerosol of HTP has been found to be slightly lower than that of conventional cigarettes, ${ }^{1617}$ but these can deliver levels of nicotine similar to conventional cigarettes according to peak plasma concentration measurements. ${ }^{17}$ The ability of HTPs to serve as a substitute for conventional cigarettes, in part, depends on the degree of nicotine dependence, which would influence the patterns of use. A randomised controlled trial showed that e-cigarettes have the potential to substitute convention cigarettes for some users. ${ }^{18}$ Higher rates of substitution might occur if products such as e-cigarettes and HTP could resemble the nicotine delivery profile of a conventional cigarette. ${ }^{19}$ Other than a clinical study among 25 cigarette smokers switching to IQOS over a short period, ${ }^{20}$ we are not aware of other assessments of nicotine dependence among HTP users that compared exclusive daily and non-daily cigarette smokers and dual HTP and cigarette users. The time-to-firstcigarette after waking up, an item from the Fagerström Test for Nicotine Dependence (FTND), has been shown to be a valid predictor of smoking cessation among conventional cigarette smokers. ${ }^{21} 22$ Therefore, we estimated and compared the prevalence of nicotine dependence using three different cut-offs of time-to-first-smoke-under 5, 15 and $30 \mathrm{~min}$-of exclusive daily cigarette, dual daily cigarette + HTP ( $\geq 1$ day), dual nondaily cigarette + HTP ( $\geq 1$ day) and exclusive HTP users, relative to exclusive non-daily cigarette users.

\section{METHODS}

\section{Data source}

We used data from the Japan 'Society and New Tobacco' Internet Survey (JASTIS), an annual internet-based cohort study that began in 2015. The purpose of JASTIS is to examine the use and perceptions of conventional tobacco products, HTPs, and electronic cigarettes, and relevant socioeconomic indicators. ${ }^{23}$ Participants were recruited from a large survey panel managed by Rakuten Insight, a major internet agency in Japan. ${ }^{23}$ The panel was drawn from a pool of 2.3 million people that considered wide-ranging socioeconomic variables, including education, housing and marital status, as defined by the Japan census. ${ }^{23}$ More in-depth details of the sampling methods for the JASTIS cohort have been described elsewhere. ${ }^{23}$ For this study, we used data from the 2019 wave of JASTIS (collected from January to February 2019) and focused on adults 25 years old and above. We restricted the analysis to participants aged 25 years and older as we also considered education (ie, avoiding collinearity), and for comparability with other studies. Initially, we had included e-cigarette users in the analysis, but the numbers were low, which was reflective of low e-cigarette use in Japan. Therefore, we further excluded the following from our analytical sample: 33 exclusive e-cigarette users, 58 dual cigarette + e-cigarette users and 32 dual $\mathrm{HTP}+\mathrm{e}$-cigarette users. Our analysis was then limited to those who smoked HTP and/or cigarettes (and no other tobacco products) for at least 1 day in the past 30 days $(n=2146)$. Figure 1 is a detailed flow chart that describes the derivation of the analytical sample.

\section{Measures}

\section{Outcome}

Nicotine dependence was assessed by time-to-first-use of tobacco in the morning, the first item from the FTND. ${ }^{21}$ The following question was asked in the survey: 'After waking up, within how much time do you smoke/use a tobacco product? The type of tobacco product does not matter.' Possible responses: within 5
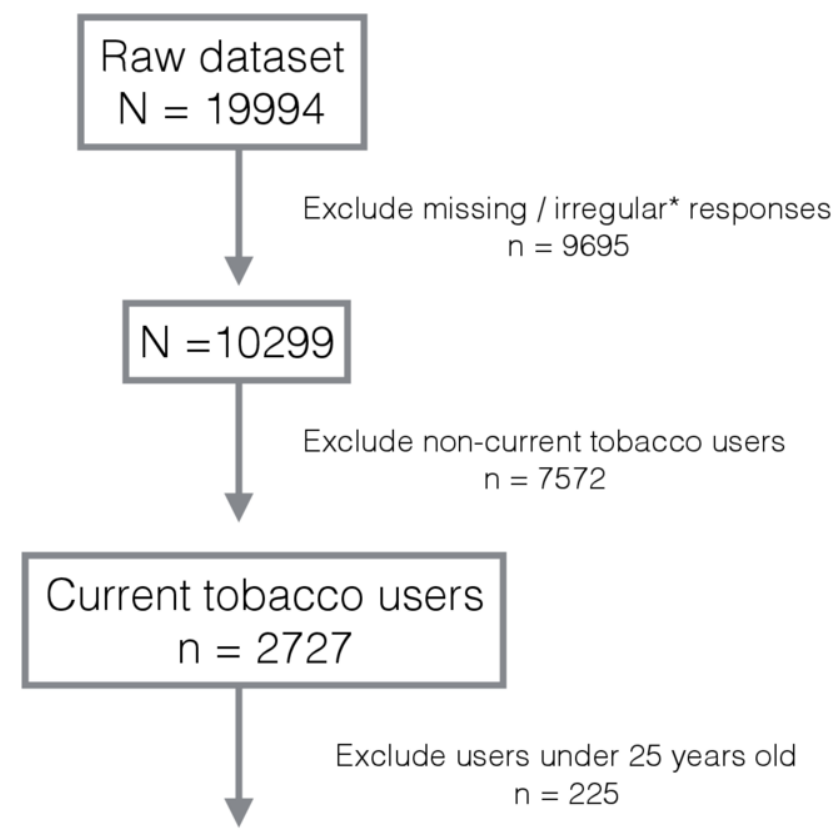

Exclude some e-cigarette users, $n=123$ (exclusive: $\mathrm{n}=33$;

dual with HTP: $n=32$; dual with cigarettes: $n=58$ )

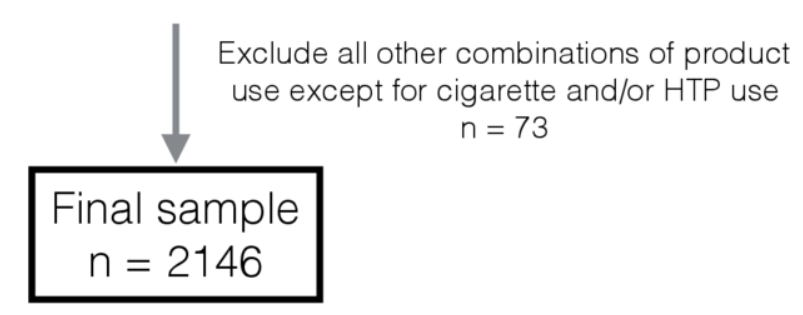

Figure 1 Data flow (*irregular responses refer to respondents who answered with the same number over an entire set of questions). HTP, heated tobacco product.

min, 6-15 min, 16-30 min, 31-60 min and more than an hour. We used the first three responses as cut-offs to define increasing severity of nicotine dependence, where 'yes' would be classified as being 'dependent': within $5 \mathrm{~min}$ (yes/no), $15 \mathrm{~min}$ (yes/ no) and $30 \mathrm{~min}$ (yes/no). See online supplemental material 1 for the wording of the survey questions, translated from Japanese to English.

\section{Exposure: definition of tobacco use}

Participants were asked the question: 'In the past 30 days, how many days did you smoke/use the following?' (please refer to online supplemental material 1 for the full list of products). Past30-day use ( $\geq 1$ day), daily past-30-day use and non-daily use in the past 30 days have been employed in other tobacco cohort studies that describe patterns of exclusive, dual product and polytobacco use. ${ }^{24-26}$ Together with the distribution of responses to the survey question (see online supplemental material 2), we categorised the following types of tobacco use, among those who reported smoking $1+$ days in the past 30 days: (1) exclusive, nondaily ( $<30$ days) cigarette; (2) exclusive HTP ( $\geq 1$ day); (3) dual HTP ( $\geq 1$ day) and non-daily cigarette; (4) dual HTP ( $\geq 1$ day) and daily (30 days) cigarette; and (5) exclusive, daily cigarette. 


\section{Statistical analyses}

Crude prevalence ratios (PRs) were calculated with log-binomial regression. However, as the multivariate models had convergence problems, we used a marginal structural binomial model to estimate standardised PRs. ${ }^{27}$ Exclusive, non-daily cigarette use was used as the referent category for the analysis since it had the lowest overall prevalence of the outcome of interest. Propensity scores and inverse probability weights were estimated using generalised boosted models, based on 3000 regression trees. ${ }^{28}$ The underlying propensity model included the following pretreatment covariates/potential confounders: age (continuous), quit attempt in the past year (yes/no), sex (male/ female), self-rated health (good/bad), self-reported mental illness (yes/no), geographical region (Hokkaido+Tohoku, KitaKanto+Tokyo-ken, Chubu+Hokuriku+Chukyo-ken, Kinki (Osaka-ken+Keihan), Chugoku+Shikoku, Kyushuu+Okinawa), educational attainment (high school, junior college, university), marital status (married, not married, widowed/ divorced), household income quartiles, living alone (yes/no), and type of housing (standalone property, private apartment, public housing/company dormitory/other). We report the standardised bias/absolute standardised mean difference for each covariate as a diagnostic to check the balance between the different tobacco use categories. ${ }^{28}$ Crude PRs of nicotine dependence by tobacco use types were compared with the results of the weighted analysis. We additionally subsetted the analytical dataset to include only exclusive, daily cigarette users, and dual $\mathrm{HTP}+$ daily cigarette users; with the former as the referent category, PRs of nicotine dependence were calculated. Exclusive HTP users were further divided by daily versus non-daily as a supplementary analysis.

All analyses were performed using R V.3.6.3. Inverse probability weights were calculated using the $\mathrm{R}$ package twang, ${ }^{29}$ which were subsequently used in weighted log-binomial models from the survey package. ${ }^{30}$

\section{RESULTS}

Table 1 shows the characteristics of the analytical sample $(\mathrm{N}=2146)$ before inverse probability weighting, with the following categories of tobacco users: $15.9 \%$ exclusive, nondaily cigarette; $14.8 \%$ exclusive HTP; $13.2 \%$ dual HTP+nondaily cigarette; $21.6 \%$ dual HTP+daily cigarette and $34.5 \%$ exclusive daily cigarette users. Notably, all exclusive HTP users were all non-current cigarette smokers (ie, did not smoke cigarettes in the past 30 days in 2019 and were not never smokers in the earlier waves of follow-up).

On average, exclusive HTP users were younger (aged 47.4 years) than exclusive cigarette users (50.2 years for non-daily and 53.1 years for daily). More dual users reported having at least one quit attempt (for conventional cigarettes) in the past 12 months $(37.1 \%$ HTP + non-daily cigarette and 31.0\% HTP +daily cigarette) compared with exclusive product users $(13.5 \%$ non-daily cigarette, $18.5 \%$ daily cigarette and $10.4 \%$ HTP). There were proportionally more women who were singleproduct users (either cigarette or HTP) compared with dual users. In terms of educational attainment, there was a higher proportion of university graduates among those with some HTP use (49.1\% exclusive HTP, 55.1\% dual HTP+ non-daily cigarette and $56.5 \%$ dual HTP+daily cigarette) compared with exclusive cigarette use (45.2\% among non-daily and 40.8\% among daily smokers). Exclusive cigarette smokers had higher proportions in the lowest income quartile (19.4\% non-daily and $21.4 \%$ daily) relative to those with some HTP use $(14.2 \%$ exclusive HTP,
14.8\% dual HTP+non-daily cigarette and 13.1\% dual HTP+daily cigarette).

Regarding nicotine dependence: when time-to-first-smoke was within 5 min of waking up, the tobacco use category with the highest proportion with nicotine dependence was exclusive, daily smokers (29.7\%), followed by dual HTP+daily cigarette (27.6\%), dual HTP+non-daily cigarette (21.2\%), exclusive, non-daily cigarette (19.9\%) and exclusive HTP users (19.8\%). The ranking changed when the cut-off was $15 \mathrm{~min}$, where the highest was instead dual HTP+daily cigarette (60.6\%) users, then exclusive daily cigarette $(57.8 \%)$, exclusive HTP $(50.0 \%)$, dual HTP+ non-daily cigarette (48.4\%) and exclusive, non-daily cigarette users (40.2\%). This ranking was the same when $30 \mathrm{~min}$ was used as the cut-off, but with higher proportions classified as being nicotine dependent across the tobacco use categories.

Baseline differences between the different categories ('populations') of tobacco use after applying inverse probability weights were 0.11 or less ( $>0.2$ is an indication of imbalance).$^{28} \mathrm{~A}$ graphical inspection of box plots of propensity scores showed sufficient overlap of the distribution of propensity scores between the categories (data not shown).

Figure 2 describes the PRs using log-binomial models standardised with inverse probability weights (for reference, crude PRs are provided in online supplemental material 3(table S3.1). Using the $5 \mathrm{~min}$ cut-off, the prevalence of nicotine dependence was the highest among exclusive, daily cigarette users $(\mathrm{PR}=1.48$, 95\% CI: 1.15 to 1.91 ), followed by dual HTP+daily cigarette $(\mathrm{PR}=1.38,95 \% \mathrm{CI}: 1.05$ to 1.82$)$, relative to exclusive, nondaily cigarette users. However, a difference in the prevalence of nicotine dependence was not detected in dual HTP + non-daily cigarette users and exclusive HTP users, relative to exclusive, non-daily cigarette users.

When the 15 min cut-off was used, nicotine dependence was higher across the different categories of tobacco use, relative to exclusive, non-daily smoking. Specifically, the prevalence was 1.38 times (95\% CI: 1.19 to 1.61 ) higher among exclusive daily cigarette users, 1.47 times (95\% CI: 1.25 to 1.72 ) higher among dual HTP+daily cigarette users, 1.24 times (95\% CI: 1.02 to 1.51) higher among dual HTP + non-daily cigarette users, and 1.23 times (95\% CI: 1.02 to 1.47 ) higher among exclusive HTP users. A similar trend can be seen when the 30 min cut-off was used to define nicotine dependence.

Analysis of the subset of daily cigarette smokers (exclusive and dual HTP) is depicted in figure 3 . Regardless of the cut-off used, there was no discernible difference of nicotine dependence between the two groups of daily cigarette users as the PRs across the different cut-offs all crossed the null. When exclusive HTP users in the main analysis (ie, figure 2) were split into daily and non-daily, the PR for exclusive non-daily HTP users crossed the null (online supplemental table 4.2).

\section{DISCUSSION}

This study examined the prevalence of nicotine dependence among different groups of tobacco users (relative to exclusive, non-daily smokers) namely: exclusive, daily cigarette; dual HTP+daily cigarette; dual HTP+non-daily cigarette and exclusive HTP users. To the best of our knowledge, this is the first paper to examine nicotine dependence among HTP users. We found that exclusive daily cigarette users and dual users of $\mathrm{HTP}+$ daily cigarettes had higher prevalence of nicotine dependence relative to exclusive non-daily cigarette smokers, across all cut-offs for time-to-first-smoke. Exclusive HTP users showed higher levels of nicotine dependence than non-daily cigarette 
Table 1 Descriptive statistics by tobacco use categories $(n=2146)$

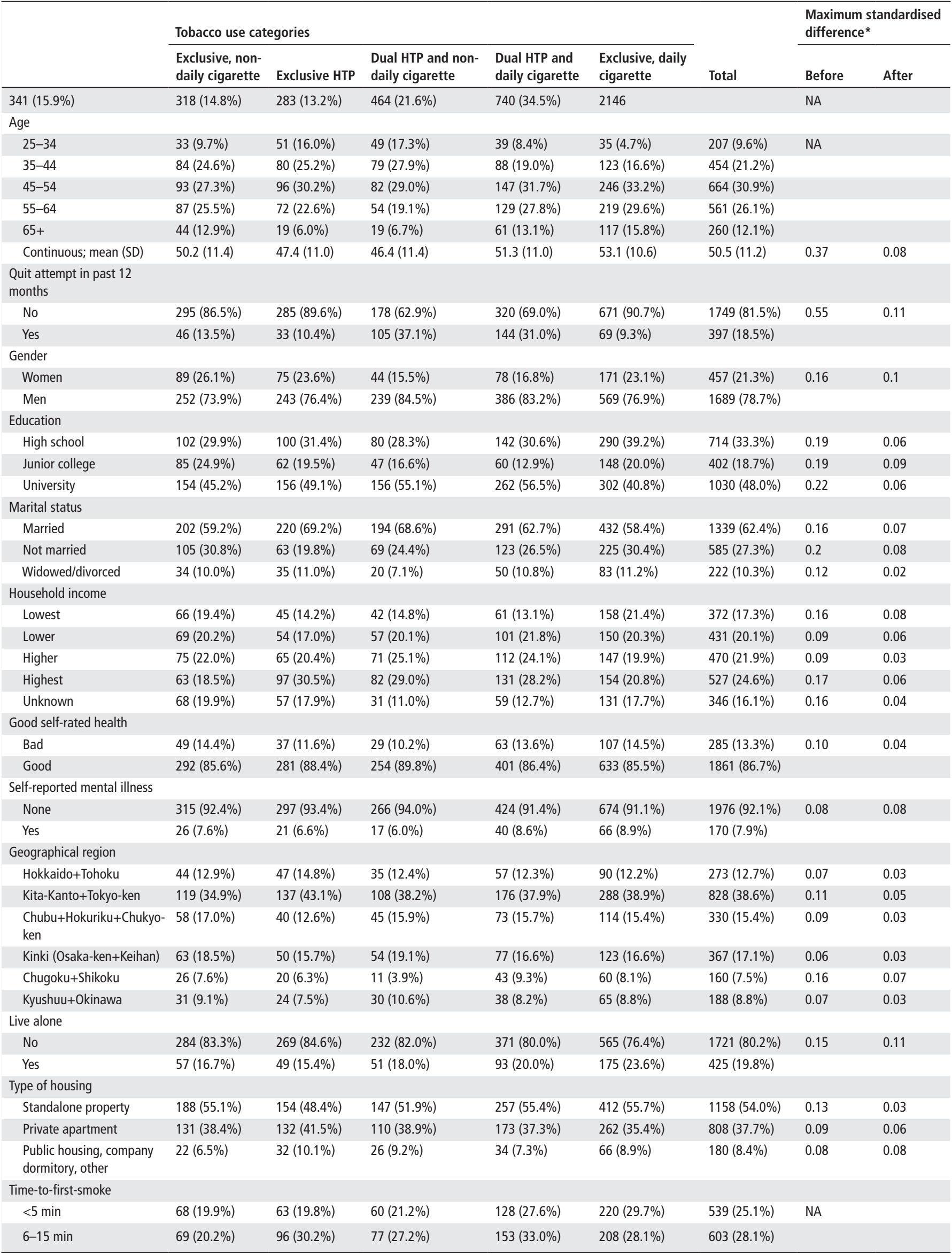




\begin{tabular}{|c|c|c|c|c|c|c|c|c|}
\hline & \multicolumn{5}{|c|}{ Tobacco use categories } & \multirow[b]{2}{*}{ Total } & \multicolumn{2}{|c|}{$\begin{array}{l}\text { Maximum standardised } \\
\text { difference* }\end{array}$} \\
\hline & $\begin{array}{l}\text { Exclusive, non- } \\
\text { daily cigarette }\end{array}$ & Exclusive HTP & $\begin{array}{l}\text { Dual HTP and non- } \\
\text { daily cigarette }\end{array}$ & $\begin{array}{l}\text { Dual HTP and } \\
\text { daily cigarette }\end{array}$ & $\begin{array}{l}\text { Exclusive, daily } \\
\text { cigarette }\end{array}$ & & Before & After \\
\hline $16-30 \mathrm{~min}$ & $40(11.7 \%)$ & $50(15.7 \%)$ & $37(13.1 \%)$ & $72(15.5 \%)$ & $120(16.2 \%)$ & $319(14.9 \%)$ & & \\
\hline $31-60 \mathrm{~min}$ & $48(14.1 \%)$ & $36(11.3 \%)$ & $48(17.0 \%)$ & $65(14.0 \%)$ & $93(12.6 \%)$ & $290(13.5 \%)$ & & \\
\hline$>1$ hour & $116(34.0 \%)$ & $73(23.0 \%)$ & $61(21.6 \%)$ & 46 (9.9\%) & $99(13.4 \%)$ & $395(18.4 \%)$ & & \\
\hline \multicolumn{9}{|l|}{ Under $5 \mathrm{~min}$} \\
\hline No & $273(80.1 \%)$ & $255(80.2 \%)$ & $223(78.8 \%)$ & $336(72.4 \%)$ & $520(70.3 \%)$ & 1607 (74.9\%) & NA & \\
\hline Yes & $68(19.9 \%)$ & $63(19.8 \%)$ & $60(21.2 \%)$ & $128(27.6 \%)$ & $220(29.7 \%)$ & $539(25.1 \%)$ & & \\
\hline \multicolumn{9}{|c|}{ Under $15 \mathrm{~min}$} \\
\hline No & $204(59.8 \%)$ & $159(50.0 \%)$ & $146(51.6 \%)$ & $183(39.4 \%)$ & $312(42.2 \%)$ & $1004(46.8 \%)$ & NA & \\
\hline Yes & $137(40.2 \%)$ & $159(50.0 \%)$ & $137(48.4 \%)$ & $281(60.6 \%)$ & $428(57.8 \%)$ & $1142(53.2 \%)$ & & \\
\hline \multicolumn{9}{|c|}{ Under $30 \mathrm{~min}$} \\
\hline No & $164(48.1 \%)$ & $109(34.3 \%)$ & $109(38.5 \%)$ & $111(23.9 \%)$ & $192(25.9 \%)$ & $685(31.9 \%)$ & NA & \\
\hline Yes & $177(51.9 \%)$ & $209(65.7 \%)$ & $174(61.5 \%)$ & $353(76.1 \%)$ & $548(74.1 \%)$ & $1461(68.1 \%)$ & & \\
\hline
\end{tabular}

* Maximum absolute standardised mean difference before and after inverse probability weighting.

†The groupings comprise of the following prefectures:.'Hokkaido+Tohoku': Hokkaido, Aomori, Iwate, Miyagi, Akita, Yamagata,Fukushima,'Kita-Kanto+Tokyo-ken': Ibaraki, Tochigi, Gunma, Saitama, Chiba, Tokyo,Kanagawa, 'Chubu+Hokuriku+Chukyo-ken': Niigata, Toyama, Ishikawa, Fukui, Yamanashi, Nagano, Gifu, Shizuoka, Aichi, Mie, 'Kinki (Osaka-ken+Keihan)': Shiga, Kyoto, Osaka, Hyogo, Nara, Wakayama, 'Chugoku+Shikoku': Tottori, Shimane, Okayama, Hiroshima, Yamaguchi,Tokushima, Kagawa, Ehime, Kochi, 'Kyushuu+Okinawa': Fukuoka, Saga, Nagasaki, Kumamoto, Oita, Miyazaki,Kagoshima, Okinawa.

HTP, heated tobacco product; NA, not available.

smokers when using the 30 and 15 min cut-offs, but not when using the 5 min cut-off. A similar pattern is observed for dual HTP+non-daily cigarette users, relative to non-daily cigarette smokers.

The potential individual and population health effects of HTP use are still unknown. First, while lower levels of known carcinogens (TNSAs and volatile organic compounds) have been detected in HTP aerosol relative to conventional cigarette smoke, much higher levels of HPHCs of unknown health risks have been found in HTPs. ${ }^{12}$ Furthermore, lower levels of harmful compounds do not translate to proportionally lower levels of health risk. ${ }^{31}$ Therefore, the relative risks of HTPs versus conventional cigarettes are still uncertain. Second, if HTPs did truly have lower risks compared with conventional cigarettes, and current exclusive, daily cigarette users do end up smoking

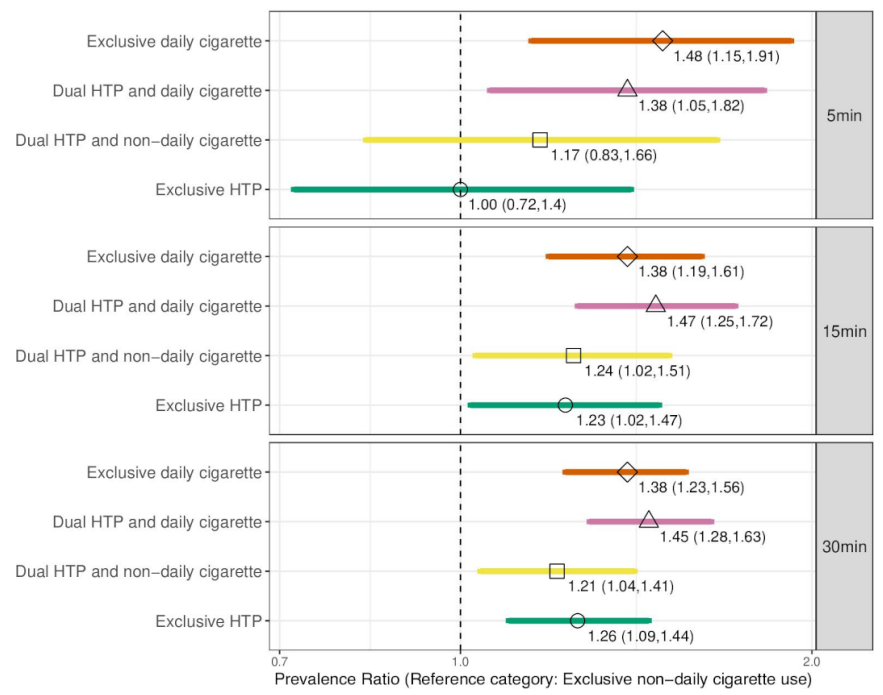

Figure 2 Nicotine dependence prevalence ratios by categories of tobacco use, and 5, 15 and 30 min cut-offs from time-to-first tobacco use after waking up ( $\mathrm{N}=2146)$. HTP, heated tobacco product. fewer cigarettes and eventually quit; or, people who would have been exclusive conventional cigarette users never end up starting smoking cigarettes as a result of using HTPs, then HTP use could result in net health gains. However, if HTP users were to sustain cigarette smoking alongside HTP use, making it more difficult to quit tobacco altogether, then HTP use could end up resulting in net harms. Similarly, if HTP use were to lead to conventional cigarette smoking in otherwise never smokers, then these would result in a net harm. Nicotine delivery and dependence are some of the factors that are likely to play a key role in the interplay between smoking and HTPs, and the eventual health impacts of HTPs.

With the rapid growth of HTP in Japan and its steady introduction around the world, it is critical to continue monitoring individual and population use of these products and their potential public health impact. Longitudinal studies of HTP use and cigarette smoking are especially needed to help us better understand the potential health consequences of HTP use and how it is affecting cigarette smoking. In particular, it is critical to determine if dual use is a transitional period, for example from exclusive smoking to exclusive HTP use, or back to exclusive smoking, or a more permanent use state. Interestingly, in

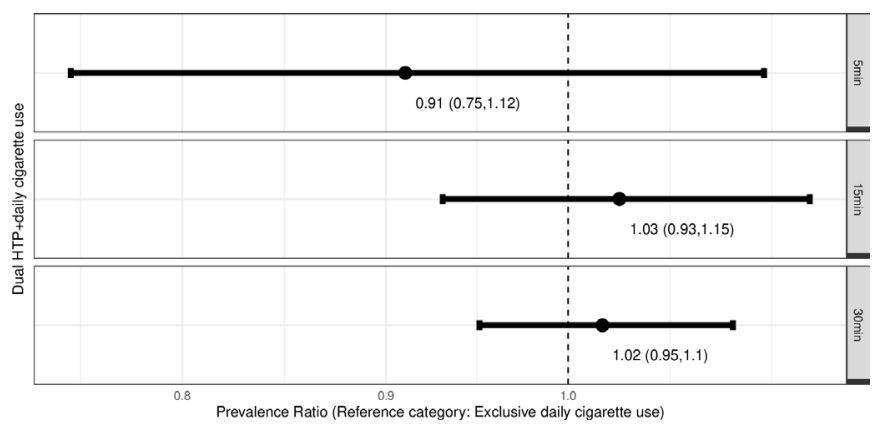

Figure 3 Nicotine dependence prevalence ratio of dual HTP and daily cigarette users by 5, 15 and 30 min cut-offs from time-to-first tobacco use after waking up ( $\mathrm{N}=1204)$. HTP, heated tobacco product. 
our sample, all exclusive HTP users were non-current cigarette smokers who reported not having smoked cigarettes the past 30 days, suggesting that switching is indeed a possibility. However, we cannot rule out an eventual relapse to cigarettes among exclusive HTP users, particularly if nicotine addiction is sustained.

While the higher nicotine dependence among daily cigarette smokers versus non-daily cigarette smokers found in this study may be unsurprising, the role of HTP is less clear. As the study design is cross-sectional in nature, we cannot assess whether a particular baseline level of nicotine dependence determined a particular pattern of tobacco use. We also could not account for a smoking history as a sizeable proportion of the participants were not asked about when they first started smoking, although current age could be thought of as an imperfect proxy for the duration smoked. Moreover, our definition of nicotine dependence was based on the first item of FTND ${ }^{21}$ which is the most informative single item to assess dependence and has been validated as a marker of dependence for conventional cigarette smoking. However, this marker has not been validated for exclusive HTP or dual use. It is possible that time-to-first product use varies by product due to environmental or other factors rather than dependence per se. For example, HTP users who might be less nicotine dependent than an exclusive non-daily, cigarette smoker might take shorter time-to-first-use because HTP can be used indoors. Similarly, product-specific use latencies (ie, timeto-use) may have differential validity as a measure of dependence in dual users. Further research would be required to account for patterns of tobacco use with HTPs and nicotine dependence assessment specific for these products. Intensity of product use would also be an important determinant of nicotine dependence. However, we did not account for the frequency of use of each product smoked per day as we could not determine the cut-offs for HTP that would be appropriate relative to conventional cigarettes. Determining an equivalence between HTP sticks with conventional cigarette sticks is an important direction for future research. Different HTPs have different nicotine concentration

\section{What this paper adds}

What is already known on this subject

- Heated tobacco products are relatively novel tobacco products with a substantial market in Japan, and are being steadily introduced around the world.

- Nicotine dependence is an important factor in tobacco addiction.

What important gaps in knowledge exist on this topic

- Little is known about nicotine dependence in heated tobacco products, and the interplay between them and conventional cigarette smoking.

- The net public health impact of heated tobacco products will depend on their patterns of use and on their ability to serve as a substitute for conventional cigarettes, which in part depends on nicotine dependence.

\section{What this paper adds}

- Using time-to-first-use to approximate nicotine dependence, we found that dual users of heated tobacco and daily cigarettes were as heavily nicotine dependent as exclusive, daily cigarette users.

- Exclusive heated tobacco users were as heavily nicotine dependent compared with non-daily cigarette users, but less dependent than dual or exclusive, daily cigarette users. and delivery, ${ }^{17}$ which may be associated with different degrees of nicotine dependence. In our analysis, we did not differentiate between different products, although most were IQOS users. Lastly, these study results may not be generalisable to other countries as the leading market of HTPs in the world is Japan where the prevalence and patterns of tobacco product use would differ as a result. Despite these limitations, this dataset is from 2019 and provides a timely and informative perspective of a relatively unknown topic.

\section{CONCLUSIONS}

Dual users of heated tobacco and daily cigarettes were as heavily nicotine dependent as exclusive daily cigarette users. Exclusive heated tobacco users were as heavily as, or potentially even more heavily nicotine dependent, compared with non-daily cigarette users, but less dependent than dual or exclusive daily cigarette users. Further studies are needed to understand the implications of the level of nicotine addiction on the patterns of HTP use and cigarette smoking, and the potential for HTPs to be an alternative to cigarettes.

Acknowledgements RM acknowledges support from the University of Michigan Rogel Cancer Centre.

Contributors YKL, RM, KK and TT contributed to the conception of the study. SO and TT acquired the data. YKL conducted the analysis and drafted the work. All authors contributed to the interpretation of data, revised the manuscript critically for important intellectual content, approved the version of the submitted manuscript, and agree to be accountable for all aspects of the work in ensuring that questions related to the accuracy or integrity of any part of the work are appropriately investigated and resolved.

Funding The study is supported by Health Labour Sciences Research Grants (20FA1005; 19FA0501; 19FA2001 and 19FA1011) and the Japan Society for the Promotion of Science (JSPS) KAKENHI Grants (18H03062).

Competing interests KK received a JMWH Bayer Grant (1 million Japanese yen) from 1 September 2017 to 31 August 2019.

\section{Patient consent for publication Not required.}

Ethics approval The study was reviewed and approved by the Research Ethics Committee of the Osaka International Cancer Institute (no. 1412175183) and the National Institute of Public Health (NIPH-IBRA\#12112).

Provenance and peer review Not commissioned; externally peer reviewed.

Data availability statement The JASTIS data can be accessed via the corresponding author, Takahiro Tabuchi, upon reasonable request.

Open access This is an open access article distributed in accordance with the Creative Commons Attribution Non Commercial (CC BY-NC 4.0) license, which permits others to distribute, remix, adapt, build upon this work non-commercially, and license their derivative works on different terms, provided the original work is properly cited, appropriate credit is given, any changes made indicated, and the use is non-commercial. See: http://creativecommons.org/licenses/by-nc/4.0/.

\section{ORCID iDs}

Yan Kwan Lau http://orcid.org/0000-0002-1612-9912

Rafael Meza http://orcid.org/0000-0002-1076-5037

Takahiro Tabuchi http://orcid.org/0000-0002-1050-3125

\section{REFERENCES}

1 Farsalinos KE, Yannovits N, Sarri T, et al. Nicotine delivery to the aerosol of a HeatNot-Burn tobacco product: comparison with a tobacco cigarette and e-cigarettes. Nicotine Tob Res 2018;20:1004-9.

2 Japan: Ministry of Health, Labor and Welfare. National health and nutrition survey 2018 summary report, 2020. Available: https://www.mhlw.go.jp/content/10900000/ 000584138.pdf

3 Philip Morris International Inc. Reports 2019 Fourth-Quarter \& Full-Year Results | Philip Morris International. Available: https://www.pmi.com/media-center/pressreleases/press-release-details/?newsld=21846 [Accessed 4 Mar 2020].

4 The Motley Fool. Philip Morris international Inc (PM) Q4 2019 earnings call transcript. Available: https://www.fool.com/earnings/call-transcripts/2020/02/06/philip-morrisinternational-inc-pm-q4-2019-earning.aspx [Accessed 4 Mar 2020] 
5 British American tobacco preliminary announcement - year ended 31 Dec 2019. Available: https://www.bat.com/group/sites/uk_9d9kcy.nsf/vwPagesWebLive/ D072TJQU/\$FILE/medMDBM7CH7.pdf?openelement [Accessed 5 Mar 2020].

6 Japan tobacco 2019 earnings report. Available: https://www.jt.com/investors/results/ forecast/pdf/2019/Full_Year/20200206_10.pdf

7 Stoklosa M, Cahn Z, Liber A, et al. Effect of IQOS introduction on cigarette sales: evidence of decline and replacement. Tob Control 2020;29:381-7.

8 Cummings KM, Nahhas GJ, Sweanor DT. What is accounting for the rapid decline in cigarette sales in Japan? Int J Environ Res Public Health 2020;17:3570.

9 Hwang JH, Ryu DH, Park S-W. Heated tobacco products: cigarette complements, not substitutes. Drug Alcohol Depend 2019;204:107576.

10 St Helen G, Jacob lii P, Nardone N, et al. IQOS: examination of Philip Morris international's claim of reduced exposure. Tob Control 2018;27:s30-6.

11 Center for Tobacco Products, Food and Drug Administration. Philip Morris products S.A modified risk tobacco product (MRTP) applications. food drug Adm, 2020. Available: https://www.fda.gov/tobacco-products/advertising-and-promotion/philip-morrisproducts-sa-modified-risk-tobacco-product-mrtp-applications [Accessed 14 Sep 2020].

12 Auer R, Concha-Lozano N, Jacot-Sadowski I, et al. Heat-Not-Burn tobacco cigarettes. JAMA Intern Med 2017:177:1050-2.

13 Leigh NJ, Palumbo MN, Marino AM, et al. Tobacco-Specific nitrosamines (TSNA) in heated tobacco product IQOS. Tob Control 2018;27:s37-8.

14 Inoue-Choi M, Liao LM, Reyes-Guzman C, et al. Association of long-term, lowintensity smoking with all-cause and cause-specific mortality in the National Institutes of Health-AARP diet and health study. JAMA Intern Med 2017;177:87.

15 Benowitz NL. Nicotine addiction. N Engl J Med 2010;362:2295-303.

16 Bekki $\mathrm{K}$, Inaba Y, Uchiyama $\mathrm{S}$, et al. Comparison of chemicals in mainstream smoke in Heat-not-burn tobacco and combustion cigarettes. J Uoeh 2017;39:201-7.

17 Simonavicius E, McNeill A, Shahab L, et al. Heat-not-burn tobacco products: a systematic literature review. Tob Control 2019;28:582-94.

18 Bullen C, Howe C, Laugesen M, et al. Electronic cigarettes for smoking cessation: a randomised controlled trial. The Lancet 2013;382:1629-37.

19 Cobb CO, Hendricks PS, Eissenberg T. Electronic cigarettes and nicotine dependence: evolving products, evolving problems. BMC Med 2015;13:119.
20 Kawamura K, Yamada K, Morioka I. [Health Effects Accompanying the Transition from Cigarettes to Heat-not-burn Tobacco: Nicotine Dependence, Nicotine Withdrawal Symptoms, and Changes in Smoking Behaviors]. Nihon Eiseigaku Zasshi 2018;73:379-87.

21 , Baker TB, Piper ME, Transdisciplinary Tobacco Use Research Center (TTURC) Tobacco Dependence, et al. Time to first cigarette in the morning as an index of ability to quit smoking: implications for nicotine dependence. Nicotine Tob Res 2007;9:555-70

22 Hatsukami DK, Stead LF, Gupta PC. Tobacco addiction. The Lancet 2008;371:2027-38.

23 Tabuchi T, Shinozaki T, Kunugita N, et al. Study Profile: The Japan "Society and New Tobacco" Internet Survey (JASTIS): A Longitudinal Internet Cohort Study of Heat-NotBurn Tobacco Products, Electronic Cigarettes, and Conventional Tobacco Products in Japan. J Epidemiol 2019;29:444-50.

24 Borland R, Murray K, Gravely S, et al. A new classification system for describing concurrent use of nicotine vaping products alongside cigarettes (so-called 'dual use'): findings from the ITC-4 Country Smoking and Vaping wave 1 Survey. Addiction 2019;114:24-34

25 Vogel EA, Prochaska JJ, Rubinstein ML. Measuring e-cigarette addiction among adolescents. Tob Control 2020;29:258-62.

26 Taylor KA, Sharma E, Edwards KC, et al. Longitudinal pathways of exclusive and polytobacco cigarette use among youth, young adults and adults in the USA: findings from the path study waves 1-3 (2013-2016). Tob Control 2020;29:s139-46.

27 Richardson DB, Kinlaw AC, MacLehose RF, et al. Standardized binomial models for risk or prevalence ratios and differences. Int J Epidemio/ 2015;44:1660-72.

28 McCaffrey DF, Griffin BA, Almirall D, et al. A tutorial on propensity score estimation for multiple treatments using generalized boosted models. Stat Med 2013;32:3388-414.

29 Ridgeway G, McCaffrey D, Morral A. Twang: toolkit for weighting and analysis of nonequivalent groups, 2017. Available: https://CRAN.R-project.org/package=twang

30 Lumley T. Survey: analysis of complex survey samples, 2019.

31 Pechacek TF, Babb S. How acute and reversible are the cardiovascular risks of secondhand smoke? BMJ 2004:328:980-3. 